\title{
INFORMATION EXCHANGE WITH COLLISION DETECTION ON MULTIPLE CHANNELS
}

\author{
Yuepeng Wang ${ }^{1}$, Yuexuan $\mathrm{Wang}^{2}$, Dongxiao $\mathrm{Yu}^{2}$, Jiguo $\mathrm{Yu}^{3}$, Francis C.M. \\ $\mathrm{Lau}^{2}$ \\ 1 School of Computer Science and Technology \\ University of Science and Technology of China \\ Hefei, 230027, P.R. China \\ wangyuep@mail.ustc.edu.cn \\ 2 Department of Computer Science, The University of Hong Kong, \\ Pokfulam, Hong Kong, P.R. China \\ amywang@hku.hk, dxyu@cs.hku.hk, fcmlau@cs.hku.hk \\ 3 School of Computer Science, Qufu Normal University \\ Rizhao, 276800, P.R. China \\ jiguoyu@sina.com
}

\begin{abstract}
Information exchange is a fundamental communication primitive in radio networks. We study this problem in multi-channel singlehop networks. In particular, given $k$ pieces of information, initially stored in $k$ nodes respectively, the task is to broadcast these information pieces to the entire network via a set of $\mathcal{F}$ channels. We develop efficient distributed algorithms for this task for the scenario where both the identities and the number $k$ of the initial information holders are unknown to the participating nodes.

Assuming nodes with collision detection, we present an efficient randomized algorithm for unrestricted information exchange, where multiple information items can be combined into a single message. The algorithm disseminates all the information items within $O\left(\frac{k}{\mathcal{F}}+\mathcal{F} \log ^{2} n\right)$ timeslots with high probability. To the best of our knowledge, this is the first algorithm that breaks the $\Omega(k)$ lower bound for unrestricted information exchange if only a single channel is available. This result establishes the superiority of multiple channels for the task of unrestricted information exchange. Moreover, for restricted information exchange, where each message can carry only one information item, we devise a randomized algorithm that completes the task in $O\left(k+\frac{\log ^{2} n}{\mathcal{F}}+\log n\right)$ timeslots. When $k$ is large, both algorithms are asymptotically optimal, as they can reach the trivial lower bounds of $\Omega\left(\frac{k}{\mathcal{F}}\right)$ and $\Omega(k)$ for unrestricted and restricted information exchange, respectively.
\end{abstract}

\section{Introduction}

Today, most wireless devices, such as those using wireless LAN or Bluetooth, can use multiple channels over their allocated radio spectrum to communicate. 
Devices using the 802.11 standard have access to around a dozen channels [1], whereas Bluetooth devices have access to around 75 [3]. A natural question then arises: How much faster can we send information around if indeed we have access to multiple communication channels? Surprisingly, despite the amount of work done so far on multiple access channels for the problem of transmission scheduling, the exact benefits of multiple communication channels for the fundamental task of information dissemination, or their characterization, remain largely unexplored.

In this paper we address this missing piece - the effects of multiple channels on the performance of information dissemination - in the theory of wireless network communication. We consider the task of information dissemination (also called information exchange) because it is the most fundamental one of all primitive operations. The task is to disseminate $k$ information items, initially stored at $k$ different nodes, to all the nodes in the network via a number of shared communication channels. As the channels are shared, collision of transmissions is a common phenomenon. When a collision occurs, none of the transmissions involved can be successful. The goal of an effective algorithm for information exchange is to minimize the time required to disseminate the information by avoiding collisions as much as possible.

\section{Our Results}

We study information exchange in a synchronized single-hop communication network, where each node can directly communicate with every other node. Single-hop networks on one hand represent our first step towards solving the problem for a wide range of networking scenarios, and on the other hand they can be practical as many ad-hoc wireless networks formed with a small physical space are most likely single-hop. The communication takes place through $\mathcal{F} \geq 2$ available channels. In each timeslot, each node can choose one of the $\mathcal{F}$ channels, and then to either listen to or transmit a message using the chosen channel. We assume that nodes can detect whether or not a collision has taken place. But nodes have no prior knowledge about which nodes possess the information items initially, nor the number $k$.

We first give an efficient distributed algorithm for unrestricted information exchange. A message is unrestricted if there is no bound on the number of information items that it can carry. Our algorithm can disseminate all information items to all nodes in $O\left(\frac{k}{\mathcal{F}}+\mathcal{F} \log ^{2} n\right)$ timeslots with high probability ${ }^{4}$. This result demonstrates the superiority of multiple channels over a single one for the task of information exchange when multiple information items can be combined into a single message. Note that in order to accomplish information exchange, each of the $k$ information item holders needs to transmit a message to at least one other node, and so if only one channel is available, we have a lower bound of $\Omega(k)$ on disseminating all information items. In addition to showing the advantage

\footnotetext{
${ }^{4}$ In this context, we say an event occurs with high probability if its probability of occurrence is $1-n^{-c}$ for a constant $c>0$.
} 
of multiple channels, we prove that our algorithm is asymptotically optimal for $k=\Omega\left(\mathcal{F}^{2} \log ^{2} n\right)$, by deriving an $\Omega\left(\frac{k}{\mathcal{F}}\right)$ lower bound valid for every unrestricted information exchange algorithm using $\mathcal{F}$ channels.

We then present a randomized algorithm for restricted information exchange where each message can only contain one information item. Our algorithm can accomplish information exchange in $O\left(k+\frac{\log ^{2} n}{\mathcal{F}}+\log n\right)$ timeslots with high probability, which improves the best known $O\left(k+\log ^{2} n\right)$ result [9] for a singlechannel case when $k$ is small. A trivial lower bound for solving information exchange is $\Omega(k)$, since in each timeslot, a node can only receive at most one message. The proposed algorithm is asymptotically optimal when $k=\Omega\left(\frac{\log ^{2} n}{\mathcal{F}}+\right.$ $\log n)$.

In the following sections, we first give an overview of the related work, in Section 3. The network models are presented in Section 4. We describe the unrestricted and restricted information exchange algorithms in Section 5 and Section 6 respectively. We conclude the paper in Section 7.

\section{Related Work}

The problem of restricted information exchange on multiple channels is not new $[15,14]$. But these works have a different objective: they target at asymptotically optimal solutions using as few channels as possible. In [15], Holzer et al. presented both randomized and deterministic asymptotically optimal algorithms for restricted information exchange when there are enough available channels. Specifically, for $k \leq \sqrt{\log n}$ and $\sqrt{\log n} \leq k \leq \frac{\log n-3}{\beta}$ with an elaborately set constant $\beta$, they gave randomized algorithms which can disseminate all information items in $\Theta(k)$ timeslots with high probability using $O\left(n^{1 / 2}\right)$ and $O\left(n^{\beta \log k / k}\right)$ channels, respectively. If there are $n$ channels available, a deterministic algorithm was proposed to accomplish information exchange in $O(\max \{k, \log n\})$ timeslots. The deterministic result was improved in [14] which achieves asymptotically optimal time bound using $O\left(n^{\log k / k}\right)$ channels when $k \leq \frac{1}{6} \log n$ and $\log ^{1+\rho}(n / k)$ channels otherwise. An $\Omega\left(n^{\Omega(1 / k)+\log _{k} n}\right)$ lower bound was also derived for any asymptotically optimal deterministic information exchange algorithm when the message size is restricted. With the assumption that nodes can listen to and receive messages from multiple channels concurrently, Shi et al. [21] gave an $O(\log k \log \log k)$ time randomized information exchange algorithm using $\Theta(n)$ channels. Furthermore, in a very recent paper [8], Daum et al. gave a randomized protocol with time complexity $O\left(k \log n+\frac{\log ^{2} n}{\mathcal{F}}+\log n \log \log n\right)$ when there are $\mathcal{F}$ available channels. In contrast, very few works addressed unrestricted information exchange on multiple channels. To the best of our knowledge, there is only one result, which is given in [8]. Their proposed randomized algorithm can accomplish information exchange in $O\left(k+\frac{\log ^{2} n}{\mathcal{F}}+\log n \log \log n\right)$ timeslots with high probability. All the above works did not try to study the benefit of collision detection when it is used in solving the information exchange problem on multiple channels. 
Information exchange on a single channel has been very well studied. There is a long list of papers addressing this problem since 1970s $[4,13,20]$. In singlechannel networks, information exchange is also known as contention resolution or $k$-selection. Previous studies mainly focus on the restricted information exchange with or without collision detection. Assuming collision detection as in here, a randomized adaptive protocol with expected running time of $O(k+\log n)$ was presented by Martel in [19]. By making use of the expected $O(\log \log n)$ selection protocol in [22], Kowalski [17] argued that the protocol in [19] can be improved to $O(k+\log \log n)$ in expectation. In [22], Willard also gave an $\Omega(\log \log n)$ expected time lower bound for fair selection protocols. When requiring high probability results, the best known randomized algorithm was given in [9], which can solve the $k$-selection problem in $O\left(k+\log ^{2} n\right)$ rounds without assuming collision detection. This protocol is asymptotically optimal for $k \in \Omega\left(\log ^{2} n\right)$ given the trivial lower bound $\Omega(k)$. Furthermore, an $\Omega(\log n)$ expected time lower bound for randomized $k$-selection protocols is implied by the result in [18] on the expected time needed to get the first message delivered without collision in a radio network. In a recent work [10], by assuming that the channel can provide feedback on whether a message is successfully transmitted, an $O(k)$ randomized protocol was proposed even without knowing $n$. However, the error probability that can be incurred by this protocol is $\frac{1}{k^{c}}$, rather than $\frac{1}{n^{c}}$. Concerning deterministic solutions, with collision detection and making use of the technique of tree algorithms, adaptive protocols for $k$-selection were presented with running time $O(k \log (n / k))$ in $[4,13,20]$. A lower bound of $\Omega\left(k \log _{k} n\right)$ is shown in [12] for this class of protocols. Furthermore, oblivious protocols in which the sequence of transmissions of a node is independent of the received messages have also been studied in $[16,7,17]$. There are also some other work focusing on dynamic packet arrivals, e.g., in a stochastic model [11], in adversarial queuing models $[2,5,17]$, and message arrivals determined by an adversary [23].

\section{Model and Preliminaries}

Consider a single-hop network with $n$ nodes, i.e., each pair of nodes can directly communicate with each other. There are $\mathcal{F} \geq 2$ available channels. We use $[\mathcal{F}]:=\{1,2, \cdots, \mathcal{F}\}$ to denote these channels. Time is synchronized and divided into slots, and the nodes start executing the algorithm at the same time. In each timeslot, each node can select one of the $\mathcal{F}$ channels and use this channel to either listen or transmit. When a node $v$ listens to a channel $C$, it can receive a message if and only if there is only one node transmitting on channel $C$. When two or more nodes transmit on the same channel, a collision occurs and none of these transmissions can be successful. Specifically, a node that operates on a channel in a given timeslot learns nothing about events on other channels. It is assumed that nodes can detect collisions, i.e., they can distinguish between collision and silence. Note that with collision detection, a transmitting node can know whether the transmission succeeds or not. 
We next introduce some definitions and concrete assumptions about the information exchange problem.

Definition 1 (Information Exchange). In a wireless network consisting of $n$ nodes, there are a subset of $k \leq n$ nodes, each of which is given a distinct piece of information. The information exchange problem is to disseminate all these $k$ information items to every node in the network in the fewest timeslots.

It is assumed that the subset of nodes which are assigned information items is determined by an adversary before the first time slot. We also assume that nodes have no knowledge about the number $k$, nor the subset of nodes possessing the information items. The only prior knowledge given to nodes is a polynomial upper bound on the network size $n$. As shown in the subsequent sections, an polynomial estimate only affects the time complexity of the proposed algorithms by a constant factor, so we simply use $n$ to denote the estimate. It is not difficult to provide nodes with this estimate in reality.

We study two types of information exchange: unrestricted and restricted. In unrestricted information exchange, a node can transmit a message containing multiple information items in each timeslot. In the restricted case, messages are of bounded size: precisely, we assume that each message can only carry one information item.

Before concluding this section, we state the following lemmas which will be useful later.

Lemma 1. For $i=1,2, \cdots, n, x_{i} \in\left[0, \frac{1}{2}\right]$, it holds that

$$
\left(\frac{1}{4}\right)^{\sum_{i=1}^{n} x_{i}} \leq \prod_{i=1}^{n}\left(1-x_{i}\right) \leq\left(\frac{1}{e}\right)^{\sum_{i=1}^{n} x_{i}}
$$

Lemma 2. [Chernoff Bound] For a parameter $a>0$, let $X_{1}, \ldots, X_{n}$ be independent or negatively associated non-negative random variables with $X_{i} \leq a$. Let $X=X_{1}+\cdots+X_{n}$ and $\mu=E[X]$. For $\delta>0$, it holds that

$$
\operatorname{Pr}(X \geq(1+\delta) \mu) \leq\left(\frac{e^{\delta}}{(1+\delta)^{1+\delta}}\right)^{\mu / a}
$$

Furthermore, for every $\delta \in(0,1)$,

$$
\operatorname{Pr}(X \leq(1-\delta) \mu) \leq\left(\frac{e^{-\delta}}{(1-\delta)^{1-\delta}}\right)^{\mu / a} \leq e^{-\delta^{2} \mu / 2 a}
$$

\section{Unrestricted Information Exchange Algorithm}

In this section, we present a distributed algorithm for unrestricted information exchange. We use the notation $F=\mathcal{F}-1$. 


\subsection{Algorithm}

In the algorithm, the information items are first collected to a small number of nodes through the first $F$ channels, and then these nodes would disseminate the information to all the other nodes by transmitting on the $F+1$-th channel. In particular, after the algorithm has started, each active node (possessing an information item) tries to send its message to other active nodes through a channel selected from the first $F$ channels. Once a node has successfully sent a message that contains its own information item and perhaps also other received information items to other active nodes, it becomes inactive and only listens. While a node finds that it is the only one choosing a particular channel in the round, it starts broadcasting its messages to all other nodes on the $F+1$-th channel. Since each node only becomes inactive after transmitting its information to other active nodes or broadcast a message to all others, all information items will finally get broadcast on channel $F+1$, which ensures the correctness of the algorithm.

During the algorithm, nodes may stay in four states: the information collection state $C$, the broadcast state $B$, the adjusting state $A$ and the inactive state $I$. Initially, the state of nodes storing information items is set as $C$, and others stay in state $I$ during the algorithm execution. Nodes in state $I$ do nothing except listening on channel $F+1$. The algorithm execution is divided into phases. A phase consists of $\Theta(\log n)$ rounds which contains two timeslots each. Between any two consecutive phases, there is an extra timeslot, called the adjusting timeslot, which is set for adjusting the transmission probability of nodes in state $C$. We next introduce the operations in states $C, B$ and $A$ in more detail. The pseudo-code is given in Algorithm 1.

Information Collection State $C$ : State $C$ is when a small number of nodes would collect their information items. At the beginning of the algorithm, all active nodes (that have a piece of information) are in state $C$. In each round of each phase, with probability $1 / 2$, each node in state $C$ selects channel $F+1$ on which it listens for receiving messages transmitted on this channel. With the remaining probability, nodes select an operating channel from the first $F$ channels uniformly at random. In the first timeslot of the round, nodes transmit with specified probability. In the second timeslot of the round, nodes transmits on the selected channel. The second timeslot is to determine whether there are multiple nodes in a channel or not, which is the condition for a node to change from state $C$ to state $B$, which we will describe later. In the adjusting timeslot, all nodes in state $C$ listen on channel $F+1$.

The transmission probability of nodes in state $C$ is initially set as $\frac{F}{2 N}$. If there is no node in state $A$ transmitting on channel $F+1$ in the adjusting timeslot after each phase, all nodes in state $C$ double their transmission probability, or otherwise they would leave the transmission probability unchanged. The transmission probability will no longer be changed after it is increased to $1 / 2$.

When a node in state $C$ receives a message, it combines the received information items and its original message into a new one, then transmits the updated message during subsequent execution of the algorithm. 
Once a node $v$ in state $C$ transmits its message successfully on the selected channel in the first timeslot of a round, $v$ will change its state to $B$ or $I$ according to whether a collision is detected or not in the subsequent timeslot. If there is a collision, i.e., other nodes in state $C$ which have received $v$ 's message selecting the same channel, $v$ enters state $I$. Otherwise, $v$ enters state $B$. After each phase, nodes that received at least $12 \log n$ messages in the last phase transit to state $A$ in the adjusting timeslot.

Adjusting State $A$ : In each adjusting timeslot, nodes in state $A$ transmit on channel $F+1$ and then change its state to state $C$ in the subsequent phase.

Broadcast State $B$ : Each node in state $B$ transmits on channel $F+1$ with probability $\frac{1}{4 c_{l} F \log n}$ for $\omega F \log ^{2} n$ timeslots, where $c_{l}$ is a constant defined in Algorithm 1 and $\omega$ is a large enough constant ensuring high probability results. With probability $1-\frac{1}{4 c_{l} F \log n}$, each node listens on channel $F+1$.

\subsection{Analysis}

We show below the correctness and the efficiency of Algorithm 1. Specifically, our algorithm can accomplish information exchange in $O\left(\frac{k}{\mathcal{F}}+\mathcal{F} \log ^{2} n\right)$ timeslots with high probability. It is also proved that any information exchange algorithm needs $\Omega\left(\frac{k}{\mathcal{F}}\right)$ timeslots even if collision detection is assumed. This lower bound shows the asymptotic optimality of the proposed algorithm for large $k$.

We use $C, A, B$ to denote the set of nodes in the corresponding states, and $P_{C}$ and $P_{B}$ to denote the sum of transmission probabilities of nodes in state $C$ and $B$, respectively.

We start the proof by first showing that the probability adjusting strategy in Algorithm 1 ensures that $P_{C}$ can be upper bounded for a sufficiently long time which is crucial for bounding the time the nodes spend in state $C$.

Lemma 3. In the first $O\left(n^{2}\right)$ rounds of the algorithm's execution, with probability $1-O\left(n^{-1}\right), P_{C} \leq F / 2$.

Proof. Assume that $t$ is the first round such that the lemma does not hold. Since the transmission probability may only be increased in adjusting timeslots, $t$ must be the first round of a certain phase. Let this phase be phase $i$. By the initial transmission probability setting for nodes in state $C, i>1$. In the adjusting timeslot before phase $i$, the transmission probability can be at most doubled. The transmission probability is in the interval $\left(\frac{F}{4}, \frac{F}{2}\right]$ during phase $i-1$. Next we lower bound the number of messages which a node in $C$ can receive on the first $F$ channels during phase $i-1$.

In each round of phase $i-1$, a node $v$ selects a channel from the first $F$ channels uniformly at random with a total probability $1 / 2$. In a round $j$ during phase $i-1$, assume that $v$ chooses channel $k$. Denote $P_{r}$ as the probability that 


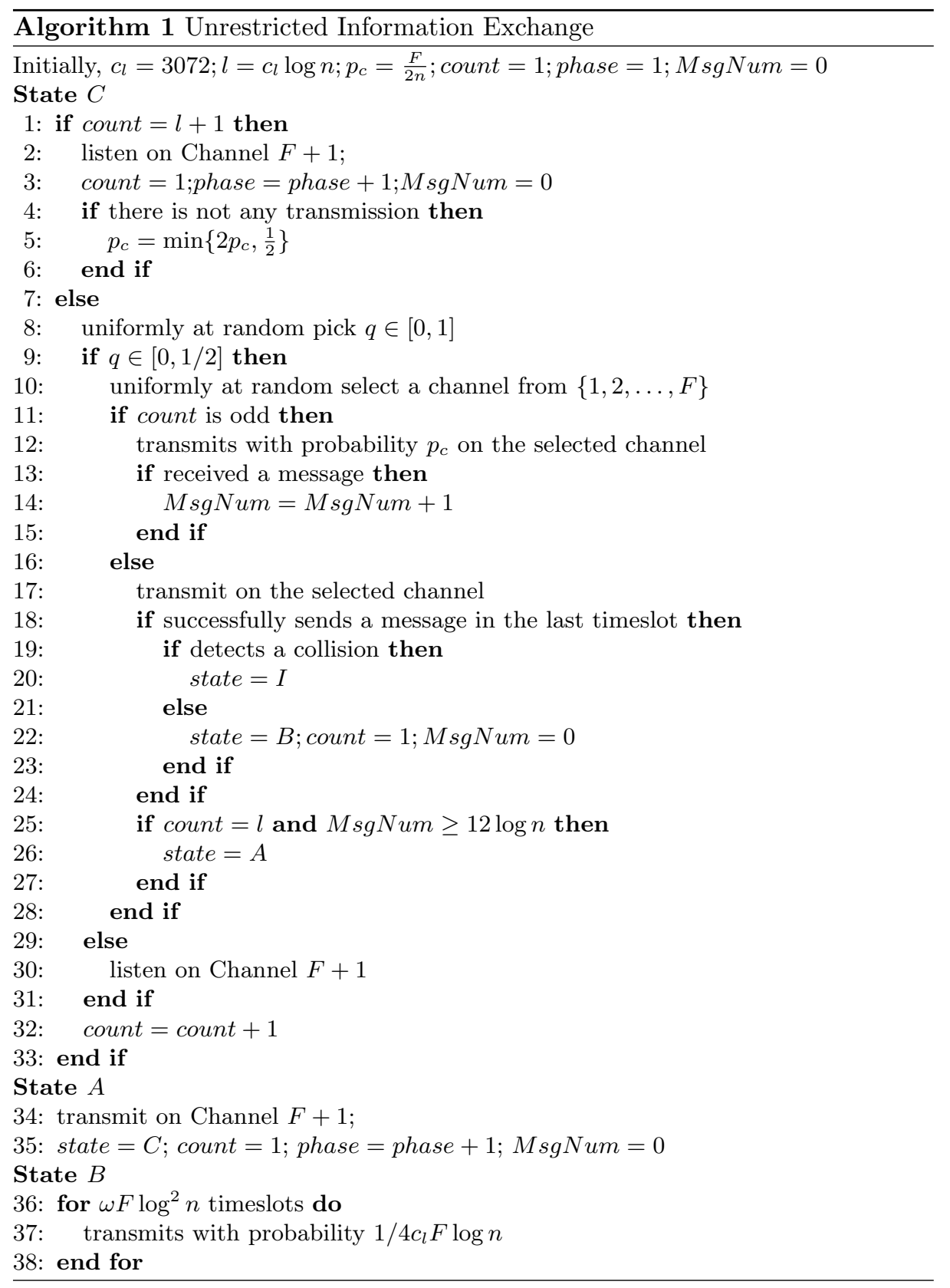


$v$ receives a message in round $j$. Then

$$
\begin{aligned}
P_{r} & =\left(1-p_{c}\right) \sum_{u \in C \backslash\{v\}} p_{c} \cdot \frac{1}{2 F} \cdot \prod_{w \in C \backslash\{u, v\}}\left(1-p_{c} \cdot \frac{1}{2 F}\right) \\
& \geq \frac{1}{2} \sum_{u \in C \backslash\{v\}} p_{c} \cdot \frac{1}{2 F} \cdot\left(\frac{1}{4}\right)^{\sum_{w \in C} p_{c} \cdot \frac{1}{2 F}} \\
& \geq \frac{1}{4} \sum_{u \in C} p_{c} \cdot \frac{1}{2 F} \cdot\left(\frac{1}{4}\right)^{\frac{F}{2} \cdot \frac{1}{2 F}} \\
& \geq \frac{1}{64}
\end{aligned}
$$

In other words, in each round of phase $i-1$, with constant probability, $v$ will receive a message. In expectation, $v$ can receive $24 \log n$ messages. By the Chernoff bound in Lemma 2, during phase $i-1, v$ can receive at least $12 \log n$ messages with probability $1-n^{-3}$. Then in the adjusting timeslot after phase $i-1, v$ joins state $A$ and transmits on channel $F+1$. All nodes in $C$ does not change the transmission probability in the subsequent phase. So in round $t$, $P_{C} \leq \frac{F}{2}$ with probability $1-n^{-3}$, which contradicts the assumption on $t$. None of the first $O\left(n^{2}\right)$ rounds are the first violating one with probability $1-O\left(n^{-1}\right)$.

For nodes in state $C$, we call a phase an increasing one if the transmission probability is doubled after that, and otherwise an unchanging phase. In the following lemma, we present a sufficient condition for a phase to be increasing, with which we can start bounding the time for nodes in state $C$.

Lemma 4. For a phase during which $P_{C} \leq \frac{F}{128}$, it is an increasing phase with probability at least $1-n^{-2}$.

Proof. Assume that phase $i$ satisfies the condition. We only need to show that there is not any node joining state $A$ after phase $i$, i.e., each node in $C$ receives less than $12 \log n$ messages during phase $i$. Consequently, after phase $i$, the transmission probability of nodes in $C$ is doubled, which completes the proof.

In each round of phase $i$, the probability of a node $v$ in state $C$ receiving a message is

$$
\left(1-p_{c}\right) \sum_{u \in C \backslash\{v\}} p_{c} \cdot \frac{1}{2 F} \cdot \prod_{w \in C \backslash\{u, v\}}\left(1-p_{c} \cdot \frac{1}{2 F}\right) \leq P_{C} \cdot \frac{1}{2 F} \leq \frac{1}{256} .
$$

Then during phase $i$, node $v$ can receive $6 \log n$ messages in expectation. Using the Chernoff bound in Lemma 2, the number of messages $v$ received is less than $12 \log n$ with probability $1-n^{-3}$. Each node receives less than $12 \log n$ messages during phase $i$ with probability $1-n^{-2}$. In other words, with probability $1-n^{-2}$, there will not be a node joining state $A$ after phase $i$, and nodes in $C$ will double the transmission probability. 
We next bound the time that nodes spend in state $C$. Let $i_{1}$ be the first phase at the beginning of which $P_{C} \geq \frac{F}{128}$. Let $i_{2}$ be the first phase at the beginning of which $|C| \leq 3 c_{l} F \log n$. We divide the execution of the algorithm for nodes in state $C$ into two stages by $i_{2}$. Stage 1 consists of phases from the beginning to phase $i_{2}-1$ and Stage 2 consists of the rest.

Lemma 5. With probability $1-O\left(n^{-1}\right)$, Stage 1 takes at most $O\left(\frac{k}{F}+\log ^{2} n\right)$ rounds.

Proof. Let $j_{1}=\min \left\{i_{1}-1, i_{2}-1\right\}$. Then we can prove the following result.

Claim. With probability $1-O\left(n^{-1}\right), j_{1} \in O(\log n)$.

Proof. By the definition of $j_{1}$, in each round of a phase $j$ with $j<j_{1}, P_{C}<\frac{F}{128}$ and there are at least $3 c_{l} F \log n$ nodes in $C$. Then by Lemma 4 , a phase $j$ with $j<j_{1}$ is an increasing phase with probability $1-n^{-2}$. In other words, after phase $j$, the transmission probability of nodes in state $C$ is doubled. Furthermore, since at the beginning of phase $j,|C| \geq 3 c_{l} F \log n$, and at most $c_{l} F \log n$ nodes in $C$ change their states in phase $j, P_{C}$ can be decreased at most by a factor of $\frac{1}{3}$. Denote $P_{C}^{i}$ as the sum of transmission probability of nodes in $C$ at the beginning of a phase $i$. Then, after phase $j, P_{C}^{j+1} \geq \frac{2}{3} P_{C}^{j} \cdot 2=\frac{4}{3} P_{C}^{j}$. Thus, with probability $1-O\left(n^{-1}\right)$, after at most $O(\log n)$ phases, either the number of nodes in $C$ decreases to less than $3 c_{l} F \log n$, or $P_{C}$ will exceed $\frac{F}{128}$, which completes the proof.

If $i_{2} \leq i_{1}$, the lemma has been proved by the above claim. We assume that $i_{1}<i_{2}$. We divide Stage 1 into two substages by $i_{1}$. Substage 1 consists of phases from the beginning to phase $i_{1}-1$ and Substage 2 consists of the rest phases in Stage 1. By the above claim, Substage 1 contains at most $O\left(\log ^{2} n\right)$ timeslots with probability $1-O\left(n^{-1}\right)$. So we only need to bound the time for Substage 2. Before that, we prove a crucial claim which shows a lower bound for $P_{C}$, as follows.

Claim. In the first $O(n)$ phases of Substage 2, with probability $1-O\left(n^{-2}\right)$, $P_{C} \geq \frac{F}{288}$.

Proof. Assume that in Substage 2, phase $j$ is the first one during which there is a round such that $P_{C}<\frac{F}{288}$. By the definition of $i_{2}$, it is easy to see that in each phase of Substage $2, P_{C}$ can be decreased by at most a factor of $\frac{1}{3}$, by which it is easy to get that $j>i_{1}$, since in each round of phase $i_{1}, P_{C} \geq \frac{F}{192}$. We can also get that in each round of phase $j-1, P_{C}<\frac{F}{128}$. Phase $j-1$ is an increasing phase with probability $1-n^{-2}$ by Lemma 4 . Note that $P_{C} \geq \frac{F}{288}$ in each round of phase $j-1$, since phase $j$ is the first one violating the lower bound on $P_{C}$. Thus, with probability $1-n^{-2}$, in any round of phase $j, P_{C} \geq 2 \cdot \frac{F}{288} \cdot \frac{2}{3}>\frac{F}{288}$, which contradicts the assumption of phase $j$. With probability $1-O\left(n^{-1}\right)$, any one of the first $O(n)$ phases in Substage 2 is not the first one violating the lower bound on $P_{C}$.

With the above claim, we are ready to bound the time for Substage 2. 
Claim. With probability $1-O\left(n^{-1}\right), i_{2}-i_{1} \in O\left(\frac{k}{F \log n}\right)$.

Proof. By Lemma 3 and the above claim, in any round of the first $O(n)$ phases in Substage 2 (if Substage 2 contains so many phases), $P_{C} \in\left[\frac{F}{288}, \frac{F}{2}\right]$ with probability $1-O\left(n^{-1}\right)$. With this bound in hand, we next show that in each phase of Substage 2, the number of nodes in $C$ is decreased by $\Omega(F \log n)$.

In a round $r$ of a phase $j$ with $i_{1} \leq j \leq \min \left\{i_{1}+O(n), i_{2}-1\right\}$, the expected number of nodes in $C$ transiting to state $B$ or $I$ is

$$
\begin{aligned}
N_{s} & =\sum_{v \in C} \frac{1}{2} p_{c} \prod_{w \in C \backslash\{v\}}\left(1-\frac{1}{2 F} \cdot p_{c}\right) \\
& \geq \sum_{v \in C} \frac{1}{2} p_{c} \cdot\left(\frac{1}{4}\right)^{\sum_{w \in C} \frac{p_{c}}{2 F}} \\
& \geq \frac{1}{4} \sum_{v \in C} p_{c} \\
& \geq \frac{F}{1152}
\end{aligned}
$$

During phase $j$, the number of nodes in $C$ is decreased by at least $F \log n$. Using the Chernoff bound, the decreasing number is at least $\Omega(F \log n)$ with probability $1-n^{-2}$. With probability $1-n^{-1}$, after at most $O\left(\frac{k}{F \log n}\right)$ phases in Substage 2, the number of nodes in state $C$ decreases to be less than $3 c_{l} F \log n$, i.e., $i_{2}-i_{1} \in O\left(\frac{k}{F \log n}\right)$. This result is obtained based on Lemma 3 and the above claim. Considering the error probability of these two results and by the union bound, the claim is proved.

Since each phase contains $\Theta(\log n)$ timeslots, the lemma is proved by above claims.

In the following Lemma, we bound the time needed for Stage 2 .

Lemma 6. With probability $1-O\left(n^{-1}\right)$, Stage 2 takes $O\left(F \log n+\log ^{2} n\right)$ timeslots.

Proof. At the beginning of Stage 2, it is easy to see that there are at most $4 c_{l} F \log n$ nodes in $C$. During each unchanging phase of Stage 2, $\Omega(\log n)$ nodes in $C$ transit to state $B$ or $I$. So there are at most $O(F)$ unchanging phases. After $O(F+\log n)$ phases, each node in $C$ will have constant transmission probability $1 / 2$. In each round of the subsequent phase, by noting that Lemma 3 still holds, the probability of a node $v$ in state $C$ sending a message successfully on the selected channel is $\frac{1}{2} p_{c} \prod_{u \in C \backslash\{v\}}\left(1-\frac{1}{2 F} p_{c}\right) \geq \frac{1}{8}$. During this phase, with probability $1-n^{-1}$, each node in $C$ will transmit a message successfully and then joins state $B$ or $I$. Combining everything, with probability $1-O\left(n^{-1}\right)$, Stage 2 takes $O\left(F \log n+\log ^{2} n\right)$ timeslots.

By Lemma 5 and Lemma 6, we have upper bounded the time needed for nodes in state $C$. To bound the running time of the algorithm, we still need to 
bound the execution time for nodes in state $B$. We first give an upper bound on the number of nodes in state $B$.

Lemma 7. There are at most $4 c_{l} F \log n$ nodes joining state $B$ with probability $1-O\left(n^{-1}\right)$.

Proof. The lemma is proved by showing that there are not nodes joining state $B$ in Stage 1. Noting that there are at most $4 c_{l} F \log n$ nodes in $C$ in Stage 2, the lemma is proved.

By the algorithm, a node enters state $B$ only if in a round, it is the only node selecting a channel from $\{1, \ldots, F\}$. In a round of Stage 1 , since there are more than $2 c_{l} F \log n$ nodes in $C$, there is at least $2 c_{l} \log n$ nodes in $C$ selecting each channel in expectation. Then for a channel $j$, using the Chernoff bound, it is easy to show that with probability $1-n^{-3}$, there are at least two nodes on $j$. This is true for all channels with probability $1-n^{-2}$, which means that with probability $1-n^{-2}$, there is not a node joining state $B$ in this round. By Lemma 5, with probability $1-O\left(n^{-1}\right)$, Stage 1 has at most $O\left(\frac{k}{F}+\log ^{2} n\right)$ rounds. Thus, with probability $1-O\left(n^{-1}\right)$, in each round of Stage 1 , there are not nodes in state $C$ joining state $B$, which completes the proof.

Lemma 8. With probability $1-O\left(n^{-1}\right)$, each node in state $B$ can successfully send its message to all other nodes on channel $F+1$ in $O\left(F \log ^{2} n\right)$ timeslots.

Proof. In each timeslot except for the adjusting timeslot, for a node $v$ in $B$, the probability that $v$ is the only transmitting node on channel $F+1$ is at least $\frac{1}{4 c_{l} F \log n} \prod_{u \in B \backslash\{v\}}\left(1-\frac{1}{4 c_{l} F \log n}\right) \geq \frac{1}{16 c_{l} F \log n}$. After $\omega F \log ^{2} n$ timeslots, by noting the listening probability of each node on channel $F+1$ being at least $1 / 2$, the probability that a node $u$ can receive the message from $v$ is at least $1-\left(1-\frac{1}{2} \cdot \frac{1}{16 c_{l} F \log n}\right)^{\omega F} \log ^{2} n \geq 1-n^{-3}$ if $\omega$ is a large enough constant. The probability that all nodes can receive the message $v$ sends with probability $1-n^{-2}$. Then by Lemma 7 , with probability $1-O\left(n^{-1}\right)$, each node in state $B$ can send its message to all other nodes.

Theorem 1. The information exchange can be accomplished after executing Algorithm 1 for $O\left(\frac{k}{\mathcal{F}}+\mathcal{F} \log ^{2} n\right)$ timeslots with probability $1-O\left(n^{-1}\right)$. Furthermore, any information exchange algorithm takes at least $\Omega\left(\frac{k}{\mathcal{F}}\right)$ timeslots.

Proof. The running time of Algorithm 1 can be obtained by Lemmas 5, 6 and 8.

By the algorithm, each node in state $C$ becomes inactive only if it transmits its message to at least one other node in state $C$. Nodes in state $C$ will finally join state $B$ if they do not become inactive, and each node in state $B$ can broadcast its message to all nodes with probability $1-O\left(n^{-1}\right)$ by Lemma 8 . So each information item can be disseminated to all nodes with probability $1-O\left(n^{-1}\right)$ during the algorithm's execution.

The lower bound is obtained by noting that each active node needs to transmit its message to at least one other node and in each timeslot, at most $\mathcal{F}$ nodes can send their messages successfully. 


\section{Restricted Information Exchange Algorithm}

In this section, we propose a randomized distributed algorithm for restricted information exchange, where each message can carry only one information item.

Again, we define $F=\mathcal{F}-1$ and denote the $F$ channels as $\{1,2, \cdots, F\}$. The $F+1$-th channel is used as a special broadcasting channel.

Without loss of generality, we assume $F \leq \log n$. Otherwise, we only use the first $\log n+1$ channels and ignore the others. For simplicity, we assume that $\log n$ is an integer and $F$ divides $\log n$.

\subsection{Algorithm}

Our algorithm uses the first $F$ channels to reduce the number of collisions on channel $F+1$. In particular, nodes first try to broadcast their messages on a channel selected from the first $F$ channels. Only those that successfully sent messages on the selected channels will broadcast on channel $F+1$. By doing so, in any timeslot, the number of nodes simultaneously transmitting on channel $F+1$ is reduced to an acceptable level, such that there is a high probability that one of these nodes can send its message to all the other nodes. The details are given in Algorithm 2.

In the algorithm, nodes may be in two states: the active state $A$ and the inactive state $I$. Initially, nodes possessing a message to broadcast enter the active state $A$, and the others stay in state $I$. After transmitting the message successfully, an active node will change its state to $I$. Nodes in state $I$ do nothing except listening on channel $F+1$ for receiving messages.

The algorithm's execution is divided into phases. Each phase consists of $l=\alpha \log n$ rounds, and each round has two timeslots, where $\alpha$ is a large enough constant for ensuring high probability results. The first slot $C$ is used for competition (competing for the chance to broadcast on channel $F+1$ ), and the second slot $B$ for broadcasting. Nodes having sent their messages successfully on selected channels in slot $C$ will transmit their messages in the subsequent slot $B$ on channel $F+1$. In slot $C$ of each round, each node in state $A$ chooses one of the first $F$ channels according to an exponential probability distribution. After choosing a channel, nodes listen with constant probability $p_{l}=\frac{3}{4}$ and broadcasts with probability $1-p_{l}$. Nodes that have successfully transmitted (with no collision) on selected channels in slot $C$ switch status to broadcast. In the subsequent slot $B$, all nodes with status broadcast broadcast their messages on channel $F+1$. At the same time, all other nodes listen on channel $F+1$. Thus, we guarantee that the message can be received by all nodes if there is exactly one node transmitting. Once a node successfully sends its message to all other nodes (with no collision), it changes its state to $I$; otherwise, it switches its status back to listen and goes on executing the algorithm.

The initial probability of choosing channel $i$ is set as $\frac{2^{i-F}}{4 n}$ for $1 \leq i \leq F$. After each phase, the selection probability is adjusted based on the number of successfully transmitting nodes on channel $F+1$ in the phase. If there are less than $12 \log n$ messages having been successfully transmitted on channel $F+1$, 


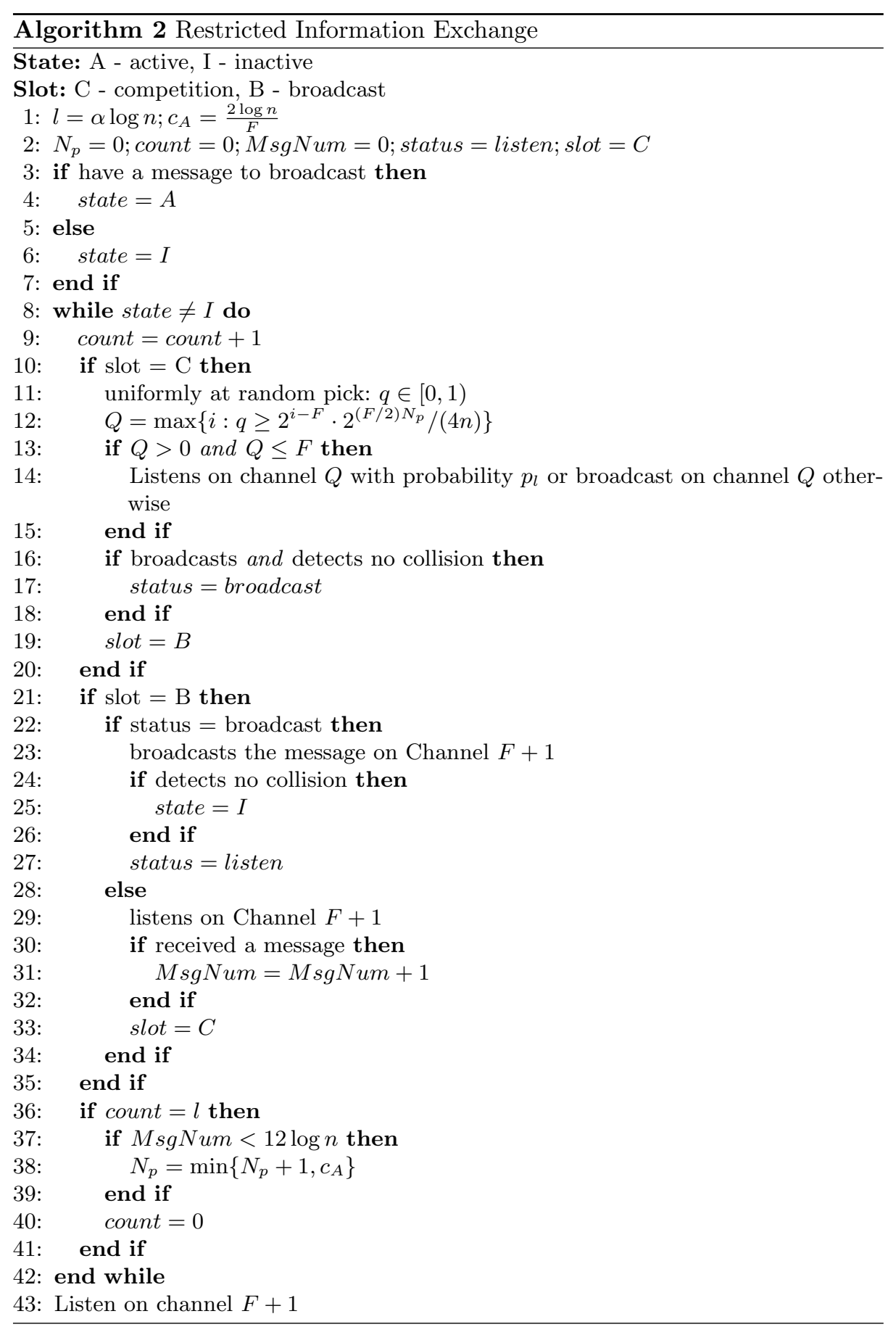


the selection probability for each channel increases by a multiplicative factor $2^{\frac{F}{2}}$; otherwise, the selection probability stays unchanged. Similar to Algorithm 1, we say a phase is increasing if the increasing condition for selection probability is satisfied, and unchanging otherwise. Then after $c_{A}=\frac{2 \log n}{F}$ increasing phases, the selection probability for channels $F, F-1, F-2, \cdots, 1$ is $\frac{1}{4}, \frac{1}{8}, \frac{1}{16}, \cdots, \frac{1}{2^{F+1}}$, respectively. Then the selection probability will not be changed any more. Note that there are $k$ messages in total which will be broadcast on channel $F+1$, so at most $O\left(\frac{k}{\log n}\right)$ increasing phases will occur.

\subsection{Analysis}

In the following we prove the correctness and efficiency of Algorithm 2.

Theorem 2. With probability $1-O\left(n^{-1}\right)$, Algorithm 2 can solve the information exchange problem in $O\left(k+\frac{\log ^{2} n}{\mathcal{F}}+\log n\right)$ timeslots.

Without confusion, we also use $A$ to denote the set of nodes in state $A$. For a channel $m$ with $1 \leq m \leq F$, we denote the probability that a node $u$ chooses $m$ as $P_{u}(m)$, and let $P_{m}=\sum_{u \in A} P_{u}(m)$ be the sum of the probability of active nodes choosing channel $m$.

We call a round successful if there is exactly one node switching status to broadcast (successfully transmitting on the selected channel) in slot $C$. Note that only nodes with status broadcast can broadcast in slot $B$, so the successful round also means there is exactly one node broadcasting in slot $B$. We give a sufficient condition for the occurrence of a successful round.

Lemma 9. If $P_{F} \in\left[\frac{1}{4}, 2^{F-2}\right]$ in a round $r$, then $r$ is successful with constant probability.

Proof. By the setting of the channel selection probability, it holds that $P_{F}=$ $2 P_{F-1}=2^{2} P_{F-2}=\cdots=2^{F-1} P_{1}$. Then we have $P_{m} \in\left[2^{m-2-F}, 2^{m-2}\right]$. Thus, there exists a channel $\lambda$ such that $P_{\lambda} \in\left[\frac{1}{4}, \frac{1}{2}\right)$. Let $A_{\lambda}^{i}$ be the event that only node $i$ transmits on channel $\lambda$. Then

$$
\begin{aligned}
\operatorname{Pr}\left(A_{\lambda}^{i}\right) & =\left(1-p_{l}\right) P_{i}(\lambda) \prod_{j \in A \backslash\{i\}}\left[1-\left(1-p_{l}\right) P_{j}(\lambda)\right] \\
& \geq\left(1-p_{l}\right) P_{i}(\lambda) \prod_{j \in A \backslash\{i\}}\left[1-\left(1-p_{l}\right) P_{j}(\lambda)\right] \\
& \geq\left(1-p_{l}\right) P_{i}(\lambda) 4^{-\left(1-p_{l}\right) P_{\lambda}}=: C_{i}^{\prime}
\end{aligned}
$$

The last inequality is by Lemma 1 . Let $A_{\lambda}$ be the event that in round $r$, there is only one node transmitting on channel $\lambda$. By the independence of channel selection of nodes, we have

$$
\begin{aligned}
\operatorname{Pr}\left(A_{\lambda}\right) & =\sum_{i \in A} \operatorname{Pr}\left(A_{\lambda}^{i}\right) \\
& \geq \sum_{i} C_{i}^{\prime}=\left(1-p_{l}\right) P_{\lambda} 4^{-\left(1-p_{l}\right) P_{\lambda}} \in \Omega(1)
\end{aligned}
$$


Similarly we define $A_{m}^{i}$ and $A_{m}$ for each channel $m$ with $1 \leq m \leq F$ and each active node $i$. By above, we have bounded the probability that there is only one node transmitting on channel $\lambda$. So in order to compute the probability that round $r$ is successful, we need to upper bound $\sum_{m \in[F] \backslash \lambda} \operatorname{Pr}\left(A_{m}\right)$. At first, we bound $\operatorname{Pr}\left(A_{m}^{i}\right)$ as follows.

$$
\begin{aligned}
\operatorname{Pr}\left(A_{m}^{i}\right) & =\left(1-p_{l}\right) P_{i}(m) \prod_{j \in A \backslash\{i\}}\left[1-\left(1-p_{l}\right) P_{j}(m)\right] \\
& =\frac{\left(1-p_{l}\right) P_{i}(m)}{1-\left(1-p_{l}\right) P_{i}(m)} \prod_{j \in A}\left[1-\left(1-p_{l}\right) P_{j}(m)\right] \\
& \leq \frac{\left(1-p_{l}\right) P_{i}(m)}{1-\left(1-p_{l}\right) P_{i}(m)} e^{-\left(1-p_{l}\right) P_{m}} \\
& \leq \frac{1-p_{l}}{p_{l}} P_{i}(m) e^{-\left(1-p_{l}\right) P_{m}}
\end{aligned}
$$

With the above upper bound on $\operatorname{Pr}\left(A_{m}^{i}\right)$, we bound $\operatorname{Pr}\left(A_{m}\right)$ as follows.

$$
\operatorname{Pr}\left(A_{\lambda}\right)=\sum_{i} \operatorname{Pr}\left(A_{\lambda}^{i}\right) \leq \frac{1-p_{l}}{p_{l}} P_{m} e^{-\left(1-p_{l}\right) P_{m}}=: C_{\lambda}
$$

Note that the function $x e^{-x} \leq e^{-1}$ for $x \geq 0$ and by the set value of $p_{l}$, we can get that $\operatorname{Pr}\left(A_{m}\right)<\frac{1}{2}$. Now we are ready to bound $\sum_{m \neq \lambda} \operatorname{Pr}\left(A_{m}\right)$.

Let $\lambda^{\prime}=\min \{\lambda+5, F\}$. Note that $P_{m+1}=2 P_{m}$, we can get the following relations on $\left\{C_{m}\right\}$.

$$
\begin{aligned}
& \frac{C_{m+1}}{C_{m}}=2 e^{-\frac{P_{m}}{4}}<\frac{1}{2}, \forall m \geq \lambda^{\prime} \\
& \frac{C_{m-1}}{C_{m}}=\frac{1}{2} e^{\frac{P_{m}}{8}}<\frac{3}{5}, \forall m \leq \lambda
\end{aligned}
$$

With the above geometric relations, we can bound $\sum_{m<\lambda} C_{m}$ by $\frac{3}{2} C_{\lambda}$ and $\sum_{m>\lambda^{\prime}} C_{m}$ by $C_{\lambda^{\prime}}$, then $\sum_{m \in[F] \backslash \lambda} \operatorname{Pr}\left(A_{m}\right)$ can be upper bounded by a constant.

$$
\begin{aligned}
\sum_{m \in[F] \backslash \lambda} \operatorname{Pr}\left(A_{m}\right) & \leq \sum_{m<\lambda} C_{m}+\sum_{m>\lambda^{\prime}} C_{m}+\sum_{m=\lambda+1}^{\lambda^{\prime}} C_{m} \\
& \leq \frac{3}{2} C_{\lambda}+C_{\lambda^{\prime}}+\sum_{m=\lambda+1}^{\lambda^{\prime}} C_{m} \\
& \leq \frac{15}{4}
\end{aligned}
$$


With the results in Inequality (5) and Inequality (7), the probability that $r$ can be lower bounded is as follows.

$$
\begin{aligned}
\operatorname{Pr}(\mathrm{r} \text { is successful }) & \geq \operatorname{Pr}\left(A_{\lambda}\right) \prod_{m \in[F] \backslash \lambda}\left(1-\operatorname{Pr}\left(A_{m}\right)\right) \\
& \geq \operatorname{Pr}\left(A_{\lambda}\right) 4^{-\sum_{m \in[F] \backslash \lambda} \operatorname{Pr}\left(A_{m}\right)} \\
& \geq \frac{1}{4} P_{\lambda} 4^{-\frac{1}{4}\left(P_{\lambda}+15\right)} \in \Omega(1)
\end{aligned}
$$

which concludes the proof.

We call a round which satisfies the condition in Lemma 9 a bounded one. In the following lemma, we show that all messages will be broadcast successfully on channel $F+1$ after at most $O(k+\log n)$ bounded rounds.

Lemma 10. All messages can be broadcast successfully on channel $F+1$ in $O(k+\log n)$ bounded rounds with probability $1-O\left(n^{-1}\right)$.

Proof. Let $X_{i}$ indicate whether the $i$-th bounded round is successful and denote by $X=\sum_{i=1}^{r} X_{i}$ the number of successful rounds in the first $r$ bounded rounds. By Lemma 9, each bounded round succeeds with constant probability. Then $E[X]=\sum_{i=1}^{r} E\left[X_{i}\right] \geq r \cdot \Omega(1)$. Let $r=\beta(k+\log n)$, where $\beta$ is a constant. Then using a standard Chernoff argument, if $\beta$ is large enough, with probability $1-O\left(n^{-1}\right)$, it is easy to show that $X \geq k$, i.e., at least $k$ nodes can successfully broadcast their messages on channel $F+1$, which means that all active nodes successfully broadcast their messages.

Before proving the final result, we need to know when the condition in Lemma 9 can be satisfied. We first show that the upper bound on $P_{F}$ holds for a long enough time after the algorithm starts.

Lemma 11. With probability $1-O\left(n^{-1}\right)$, in the first $O\left(n^{2}\right)$ rounds, $P_{F} \leq 2^{F-2}$.

Proof. The proof is similar to that for Lemma 3. We assume that round $i$ of phase $j$ is the first one in which $P_{F}$ exceeds $2^{F-2}$. It is obvious that $i$ is the first round of phase $j$. By the adjusting strategy of selection probability, $P_{F} \in\left[\frac{1}{4}, 2^{F-2}\right]$ in phase $j-1$.

By Lemma 9 , each round of phase $j-1$ is successful with constant probability. In expectation, there are at least $24 \log n$ nodes transmitting successfully on channel $F+1$ if $\alpha$ is large enough. Using the standard Chernoff Bound argument, with probability $1-n^{-3}$, there will be at least $12 \log n$ successful transmissions on channel $F+1$ in phase $j-1$. Thus, the selection probability will stay unchanged, which means that in round $i, P_{F}$ will not break the upper bound with probability $1-n^{-3}$. By the Union Bound argument, none of the first $O\left(n^{2}\right)$ rounds would be the first violating one with probability $1-O\left(n^{-1}\right)$, which completes the proof. 
With all above lemmas, we prove Theorem 2.

Proof (Proof of Theorem 2). Note that there are at most $\frac{k}{12 \log n}$ unchanging phases; after $O\left(\frac{k}{\log n}+\frac{\log n}{F}\right)$ phases, the probability of an active node choosing channel $F$ will become a constant $\frac{1}{4}$, i.e., $P_{F}$ will be at least $\frac{1}{4}$ if there still exist some active nodes. Combining the result in Lemma 11, the condition in Lemma 9 can be satisfied in the subsequent $O\left(n^{2}\right)$ rounds. By Lemma 9 , with probability $1-O\left(n^{-1}\right)$, information exchange can be completed in $O(k+\log n)$ rounds thereafter. Combining everything, the theorem is proved.

\section{Conclusion}

We presented two randomized distributed algorithms for information exchange in single-hop multiple-channel radio networks, covering respectively the unrestricted case and the restricted case. The proposed algorithms are both asymptotically optimal when the number $k$ of nodes having an information item initially is large. Furthermore, given the lower bound $\Omega(k)$ for solving unrestricted information exchange on a single channel, our first algorithm shows the superiority of multiple channels in disseminating information. An important future work is to adapt the proposed techniques to solve information exchange in multi-hop multi-channel networks.

\section{Acknowledgement}

This work was supported in part by the National Natural Science Foundation of China Grants 61073174 and 61373027, and Hong Kong RGC GRF grant 714311.

\section{References}

1. I. 802.11. Wireless LAN MAC and Physical Layer Specifications, June 1999.

2. M. A. Bender, M. Farach-Colton, S. He, B. C. Kuszmaul, and C. E. Leiserson. Adversarial contention resolution for simple channels. In Proc. of the 17th Ann. ACM Symp. on Parallel Algorithms and Architectures (SPAA), pages 325-332, 2005.

3. Bluetooth Consortium. Bluetooth Specification Version 2.1, July 2007.

4. J. Capetanakis. Tree algorithms for packet broadcast channels. IEEE Trans. Inf. Theory, IT- 25(5):505-515, 1979.

5. B.S. Chlebus, D.R. Kowalski, M.A. Rokicki. Adversarial queuing on the multipleaccess channel. In Proc. of the 25th ACM Symposium on Principles of Distributed Computing (PODC), pages 92-101, 2006.

6. B.S. Chlebus, D.R. Kowalski, and M.R. Rokicki, Average-time complexity of gossiping in radio networks, in Proceedings, 13th Colloquium on Structural Information and Communication Complexity (SIROCCO), 2006, LNCS 4056, pp. 253 - 267.

7. A. Clementi, A. Monti, and R. Silvestri. Selective families, superimposed codes, and broadcasting on unknown radio networks. In Proc. of the 12th Ann. ACM-SIAM Symp. on Discrete Algorithms (SODA), pages 709-718, 2001. 
8. S. Daum, S. Gilbert, M. Ghaffari, F. Kuhn, and C. Newport. Maximal Independent Sets in Multichannel Radio Networks. In Proceedings of the ACM Symposium on the Principles of Distributed Computing (PODC), 2013.

9. A. Fernández Anta, M. A. Mosteiro. Contention Resolution in Multiple-Access Channels: $k$-Selection in Radio Networks. In Proc. of the 16th Annual International Computing and Combinatorics Conference (COCOON), pages 378-388, 2010.

10. A. Fernández Anta, M. A. Mosteiro, J. R. Muñoz. Unbounded Contention Resolution in Multiple-Access Channels. In Proc. of the 25th International Symposium on DIStributed Computing (DISC), pages 225-236, 2011.

11. L.A. Goldberg, M. Jerrum, S. Kannan, M. Paterson. A bound on the capacity of backoff and acknowledgment-based protocols. SIAM Journal on Computing 33:313331, 2004.

12. A. Greenberg and S. Winograd. A lower bound on the time needed in the worst case to resolve conflicts deterministically in multiple access channels. Journal of the $A C M, 32: 589-596,1985$.

13. J. F. Hayes. An adaptive technique for local distribution. IEEE Trans. Comm., COM-26:1178-1186, 1978. In Proc. of the 7th ACM SIGACT/SIGMOBILE International Workshop on Foundations of Mobile Computing (FOMC), pages 69-76, 2011.

14. S. Holzer, T. Locher, Y. A. Pignolet, R. Wattenhofer. Deterministic multi-channel information exchange. In Proc. of SPAA 2012, Pages 109-120, 2012.

15. Stephan Holzer, Yvonne Anne Pignolet, Jasmin Smula, Roger Wattenhofer: Timeoptimal information exchange on multiple channels. In Prof. of FOMC 2011, Pages 69-76, 2011. In Proc. of the 13th Ann. ACM-SIAM Symp. on Discrete Algorithms (SODA), pages 697-704, 2002.

16. J. Komlòs, A. Greenberg. An asymptotically nonadaptive algorithm for conflict resolution in multiple-access channels. IEEE Trans. Inf. Theory, 31:303-306, 1985.

17. D. R. Kowalski. On selection problem in radio networks. In Proc. of 24 th Ann. ACM Symp. on Principles of Distributed Computing (SPAA), pages 158-166, 2005.

18. E. Kushilevitz, Y. Mansour. An $(D \log (N / D))$ lower bound for broadcast in radio networks. SIAM Journal on Computing, 27(3):702-712, 1998.

19. C. U.Martel. Maximum finding on a multiple access broadcast network. Inf. Process. Lett., 52:7-13, 1994.

20. V. Mikhailov, B. S. Tsybakov. Free synchronous packet access in a broadcast channel with feedback. Problemy Peredachi Inform, 14(4):32-59, 1978. Analysis. Cambridge University Press, 2005.

21. W. Shi, Q.-S. Hua, D. Yu, Y. Wang, F.C.M. Lau. Efficient Information Exchange in Single-Hop Multi-Channel Radio Networks. The 7th International Conference on Wireless Algorithms, Systems, and Applications (WASA), 2012.

22. D. E. Willard. Log-logarithmic selection resolution protocols in a multiple access channel. SIAM Journal on Computing, 15:468-477, 1986.

23. D. Yu, Q.-S. Hua, W. Dai, Y. Wang, and F.C.M. Lau. Dynamic Contention Resolution in Multiple-Access Channels. 10th International Conference on Wired/Wireless Internet Communications (WWIC 2012), 2012. 